- Published in Erkenntnis (2013)-

(http://link.springer.com/article/10.1007/s10670-013-9507-7)

\title{
Emergence, closure and inter-level causation in biological systems
}

\author{
Matteo Mossio ${ }^{1}$, Leonardo Bich ${ }^{2,}$ \& Alvaro Moreno ${ }^{2}$ \\ ${ }^{1}$ IHPST (CNRS/Université Paris I/ENS) \\ 13, rue du Four, 75006 Paris, France \\ ${ }^{2}$ Department of Logic and Philosophy of Science \\ IAS-Research Centre for Life, Mind and Society \\ University of the Basque Country \\ Avenida de Tolosa 70, 20018, Donostia-San Sebastián, Spain
}

\begin{abstract}
In this paper, we advocate the idea that an adequate explanation of biological systems requires appealing to organisational closure as an emergent causal regime. We first develop a theoretical justification of emergence in terms of relatedness, by arguing that configurations, because of the relatedness among their constituents, possess ontologically irreducible properties, providing them with distinctive causal powers. We then focus on those emergent causal powers exerted as constraints, and we claim that biological systems crucially differ from other natural systems in that they realise a closure of constraints, i.e. a higher-level emergent regime of causation such that the constituents, each of them acting as a constraint, realise a mutual dependence among them, and are collectively able to self-maintain. Lastly, we claim that closure can be justifiably taken as an emergent regime of causation, without admitting that it inherently involves whole-parts causation, which would require committing to stronger ontological and epistemological assumptions.
\end{abstract}

\section{Introduction}

Whether adequate explanations in biology require appealing to an emergent and distinctive causal regime seems to have an obvious positive answer, insofar as biological systems evolve by natural selection (Mayr, 2004, p. 31). Yet, as Wesley Salmon has pointed out (1998, p. 324), one can distinguish between etiological explanations, which tell the story leading to the occurrence of a phenomenon, and constitutive explanations, which provide a causal analysis of the phenomenon itself. Accordingly, whereas it goes without saying for etiological explanations, there seems to be no obvious answer to the question of whether a constitutive explanation of biological systems would also appeal to a distinctive regime of causation, emergent from and irreducible to that at work in physical and chemical natural systems.

During the last forty years, the idea that the constitutive organisation of biological systems does realise a distinctive regime of causation has been put forward by a number of pioneering authors like Rosen (1972, 1991), Piaget (1967), Maturana and Varela (1980), Varela et al. (1974), Varela (1979), Pattee (1972, 1973,), Ganti (1975, 2003) and recently developed in 
various fields, including Theoretical Biology (Kauffman, 2000), Biochemistry (Luisi, 2006; Cornish-Bowden et al., 2007) and Systems Biology (Hofmeyr, 2007). Broadly speaking, the common background assumption of this scientific trend, despite the differences among the various formulations, consists in an understanding of biological systems which translates into scientific terms an idea originally exposed by Immanuel Kant in his Critique of Judgement, according to which a biological system can be conceived as a natural purpose, such that:

"The parts of it produce themselves together, one from the other, in their form as much as in their binding, reciprocally, and from this causation on, produce a whole. In such a product of nature each part, at the same time as it exists throughout all the others, is thought as existing with respect to the other parts and the whole, namely as instrument (organ). It is then - and for this sole reason - that such a product, as organized and organizing itself, can be called a natural purpose” (Kant, 1987, §65, p. 287).

In this view ${ }^{1}$, then, biological systems realise a distinctive causal regime in which a set of constituents produce and maintain each other through a network of mutual interactions, such that the whole system can be said to be collectively able to self-produce and self-maintain. In the literature, this regime is usually referred to as closure (see also Mossio \& Moreno, 2010, for a recent analysis) ${ }^{2}$.

In spite of the increasing scientific and philosophical work on this notion, however, the status of closure as a distinctively biological causal regime has not yet been assessed, we hold, in adequate terms. Indeed, the very idea that closure would be 'distinctive' of the biological domain seems to require adopting a non-reductionist stance, according to which biological systems realise a regime of causation that is irreducible to those at work in other classes of natural (i.e. physical and chemical) systems. In this sense, hence, the philosophical discussion on closure is related to that on emergence, to the extent that closure can be 'irreducibly biological' only if biological systems can be shown to possess emergent causal powers. Still, existing accounts do not provide clear arguments supporting the claim that closure can be taken as an emergent causal regime.

Similarly, it is at present unclear whether or not closure involves inter-level causation. At first sight it seems obvious that closure inherently relies on the causal interplay between entities at different levels of description: the integrated activity of lower-level constituents contributes to generate the higher-level organisation, and the higher-level organisation plays a crucial role in maintaining and regenerating its own constituents, as well as controlling and regulating their behaviour and interactions. However, the appeal to inter-level causation in biological systems may oscillate between two different interpretations of the concept: on the one hand, the causal influence of an entity located at a given level of description on an external entity located at another (upper or lower) level of description; on the other hand, the causal influence of an entity, taken as a whole, on its own parts. As we will discuss, while it might seem quite obvious that closure involves inter-level causation in the first sense, a more difficult issue is whether this is also true for the second sense, which requires complying with more restrictive conceptual conditions.

\footnotetext{
${ }^{1}$ See also Weber \& Varela (2002) for a discussion of the Kantian rooting of this scientific tradition.

${ }^{2}$ It is worth noting that this meaning of 'closure' has nothing to do with Kim's one, which is at work in his argument about the "causal closure of the physical domain". According to the latter, as Kim explains, "any physical event that has a cause at time t has a physical cause at $t$. This is the assumption that if we trace the causal ancestry of a physical event, we need never go outside the physical domain”(Kim, 1993: 280).
} 
In this paper, we will advocate the view according to which an adequate constitutive explanation of biological systems requires appealing to the idea of organisational closure as an emergent regime of causation.

Our argument will be twofold. On the one hand, we will develop a theoretical justification of emergent causation, by arguing that the idea of emergent causal powers can be consistently understood in terms of constraints. We will then suggest, by providing a general characterisation, that organisational closure should be conceptualised as a network of constraints mutually interacting in a distinctive way. On the other hand, we will maintain that although the mutual relations between constraints are such that the very existence of each of them depends on their being involved in the whole organisation, an emergent closed organisation does not necessarily imply inter-level causation, be it upward or downward, in the restrictive sense of a causal relation between the whole and its own parts. Yet, as we will suggest, the appeal to inter-level causation in this sense (which is the philosophically more interesting and discussed one) may possibly be relevant for organisational closure, if the adequate conceptual justification were provided.

The structure of the paper is as follows. In section 2 we discuss one of the main philosophical challenges to the idea of emergence - Kim's exclusion argument - by focusing on the fact that it applies to a specific account of emergence formulated in terms of supervenience and irreducibility. In section 3, we recall the distinction between two dimensions of the debate about emergence - ontological irreducibility and epistemological non-derivability -, and clarify that a pertinent defence against the exclusion argument can be expressed, as we will do, exclusively in terms of irreducibility. Section 4 offers a conceptual justification of emergent properties, and argues that, because of the relatedness among their constituents, configurations possess ontologically irreducible properties, providing them with distinctive causal powers. In section 5, we focus on the specific case in which configurations exert distinctive causal powers as constraints acting on underlying physicochemical changes, and we provide a precise characterisation of their irreducible properties with respect to thermodynamic flow. Section 6 describes how constraints are distinctively at work in natural self-maintaining systems, and specifically in biological systems, in which they realise the causal regime labelled 'closure'. In particular, we argue that closure can be taken as a higherlevel emergent configuration, ontologically irreducible and provided with distinctive causal powers, including the generation of functions. Section 7 concludes the analysis, and argues that closure can be justifiably taken as an emergent biological causal regime without admitting that it inherently involves inter-level causation. Yet, the connection between closure and inter-level causation remains an open issue requiring further philosophical and theoretical investigations.

\section{The philosophical challenge to emergence}

The very idea of some distinctively biological regime of causation cannot be justified unless it can be shown that, in some way, a given entity possesses characteristic properties by virtue of which it can exert emergent causal powers. A conceptual justification of emergence seems then to be a necessary requirement for a coherent account of biological causation.

Philosophical work on emergence began during the late-nineteenth and early-twentieth centuries with the writings of the so-called "British Emergentists" (Mill, 1843; Alexander, 1920; Lloyd Morgan, 1923; Broad, 1925), and has developed considerably over recent decades. As has often been underscored, a central contribution to this debate was made by Jaegwon Kim, who developed one of the most articulated conceptual challenges to the idea of emergence (Kim, 1993; 1997; 1998; 2006). 
In a recent survey of these issues (Kim, 2006), Kim recalls what are, in his view, the two necessary ingredients of the idea of emergence, i.e. supervenience and irreducibility. Supervenience is a relation by virtue of which the emergent property of a whole is determined by the properties of, and relations between, its realisers. As he puts it (Kim, 2006, p. 550):

"Supervenience: If property $M$ emerges from properties $N_{1}, \ldots N_{n}$, then $M$ supervenes on $N_{1}, \ldots N_{n}$. That is to say, systems that are alike in respect of basal conditions, $N_{1}$, . . . $N_{n}$ must be alike in respect of their emergent properties”.

In turn, irreducibility, and more precisely, according to Kim, functional irreducibility, is expressed as follows:

"Irreducibility of emergents: Property $M$ is emergent from a set of properties, $N_{1}, \ldots$ $N_{n}$, only if $M$ is not functionally reducible with the set of the $N_{\mathrm{s}}$ as its realizer" (Kim, 2006, p. 555).

Given the account of emergent properties in terms of supervenience and irreducibility, the central issue is whether these properties may possess distinctive causal powers. In his work, Kim has developed several lines of criticisms vis-à-vis emergence. The one, which is specifically relevant here, claims that emergent properties are exposed to the threat of epiphenomenalism. Kim's argument on this matter is known as the "exclusion argument”, and it has been offered on several occasions. Very briefly, the idea is the following. If an emergent property $M$ emerges from some basal conditions $P$, and $M$ is said to cause some effect, one may ask "why cannot $P$ displace $M$ as a cause of any putative effect of M?" (Kim, 2006, p. 558). If $M$ is nomologically sufficient for whatever effect $X$, and $P$ is nomologically sufficient for $\mathrm{M}$ (because of the supervenience relation), it seems to follow that $\mathrm{P}$ is nomologically sufficient for $\mathrm{X}$, and $\mathrm{M}$ is "otiose and dispensable as a cause" of $\mathrm{X}$. As a result, invoking the causal power of emergent structures would be useless, since epiphenomenal.

The exclusion argument has crucial implications for the debate about emergence and reduction. If one admits (1) that the relation between $\mathrm{M}$ and $\mathrm{P}$ is correctly described in terms of supervenience and (2) the validity of what we will call here the principle of the inclusivity of levels ${ }^{3}$, i.e. "the idea that higher levels are based on certain complicated subsets from the lower levels and do not violate lower level laws" (Emmeche et al., 2000, p. 19), then two problematic consequences follow.

First, explanation is exposed to the danger of causal drainage. Indeed, if the causal powers of an emergent entity can be reduced to the causal powers of its constituents, and if, as it may indeed be the case, there is no 'rock-bottom' level of reality, then it seems that "causal powers would drain away into a bottomless pit, and there would not be any causation anywhere" (Campbell and Bickhard, 2011, p. 14) ${ }^{4}$. Second, if there were some scientifically justifiable

\footnotetext{
${ }^{3}$ We take here the notion of "inclusivity of levels" as analogous to Kim's "Causal Inheritance Principle” (Kim, 1993, p. 326), according to which if a property $M$ is realised when its physical realisation bases $P$ is instantiated, the causal powers of $\mathrm{M}$ are identical with the causal powers of P. By the choice of "inclusivity of levels" we want to emphasise the idea that in the natural world all causes are physical or are the result of the interaction between physical entities: no special causes (vitalist, spiritual, etc., that are not physically instantiated) are introduced at different levels, e.g. at the biological and the mental ones. It should be noted that Kim's argument also requires the Causal Closure Principle as a premise, in the sense that the ultimate reduction of an emergent property to its fundamental realisation base is possible only if the basal level is causally closed (Kim, 2003). Yet, we maintain that the validity of the inclusivity of levels does not necessarily require appealing to causal closure: emergent causal powers can be reduced to basal powers even though the latter are not shown or supposed to be closed. Consequently, the argument we develop in this paper does not depend on the Causal Closure Principle.
}

\footnotetext{
${ }^{4}$ For Kim's purposes, the exclusion argument is originally targeted at mental causation and is not supposed to imply causal drainage. As a matter of fact, Kim himself has vehemently tried to avoid causal drain drainage as the ultimate consequence of the argument of this argument in favour of reduction. In addition, on the basis of a
} 
rock-bottom level of reality (which is a far from trivial assumption) ${ }^{5}$, and causal drainage were blocked, the exclusion argument would force reductive physicalism (see Vicente, 2011 for a recent analysis). In this second case, whatever appeal to distinctively biological causal relations (such as closure itself, and related notions such as 'integration', 'control', 'regulation'...) would at best constitute heuristic tools, unless it could be demonstrated that they can adequately be reduced to physical causation or, more generally, to any 'more fundamental' regime of causation.

In both cases, the very possibility of biological explanation is menaced. An adequate justification of a distinctive regime of biological causation should be provided in order to (1) avoid the danger of endless causal drainage and (2) make biological explanation theoretically independent from the physical and chemical ones, and directly related to the specificity of biological phenomenology instead of being derived from lower level explanations, and dependent on a single physical 'theory of everything' (Laughlin and Pines, 2000).

\section{Irreducibility vs. non-derivability}

Before addressing the exclusion argument, let us make a preliminary conceptual distinction between two dimensions of the debate about emergence, i.e. irreducibility and nonderivability.

The exclusion argument challenges the status of emergent properties as causal agents of the world: how can a property be supervenient on something while being, at the same time, irreducible, and then possessing distinctive causal powers? An appropriate reply should then deal with the ontological issue of irreducibility, and justify emergent properties by showing that they are something ontologically 'new' with respect to their realisers. Irreducibility, hence, is inherently linked to ontological novelty.

Irreducibility should not be confused with the possible non-derivability of emergent properties from the emergence base, which is an epistemological issue. Non-derivability refers to the fact that given a description of the properties of the realisers, it is not possible to predict, explain or deduce the emergent properties of the whole.

As a matter of fact, most of the philosophical debate has tended to merge the two issues ${ }^{6}$, and to take both irreducibility and non-derivability as marks of emergence: emergent properties are not only irreducible but also, and crucially, non-derivable. Consider for instance the classical distinction between 'resultant' and 'emergent' properties, which is based precisely on criteria of non-derivability (or "non-deducibility", in Kim, 2006, p. 552) ${ }^{7}$. Resultant properties are aggregative properties which the whole possesses at values that the parts do not (i.e. a kilogram of sand has a mass that none of its constituents has). Emergent properties, in

commitment to the Standard Model and its bottom level of fundamental physical particles, he rejects the arguments based on the possibility of the absence of a rock-bottom level of reality. For a detailed discussion of these issues see, for example Block's criticism of Kim's reduction argument (Block, 2003) and Kim’s reply (Kim, 2003).

\footnotetext{
${ }^{5}$ The idea of a basic level with self-sufficient basic entities has been deeply questioned in microphysics, the very domain reductionist approaches appeal to as fundamental, where relational and heuristic accounts have taken place (Bitbol, 2007).

${ }^{6}$ The distinction has however been formulated, for instance by Silberstein and McGeever (1999), according to which epistemological emergence concerns models or formalisms, while ontological emergence involves irreducible causal capacities. Here, we follow this conventional distinction.

${ }^{7}$ Van Gulick (2001) refers to resultant and emergent properties as “specific value emergent” and "modest kind emergent” properties, respectively.
} 
turn, are properties of a kind that only the whole possesses, whereas the parts do not (i.e. a system can be alive, whereas none of its parts is alive). Although resultant properties can be said to be, in a general sense, irreducible to the properties of their realisers, however, they are not what British emergentists (and most contemporary authors) had in mind when speaking about emergence. In fact, when appealing to notions like 'unpredictability' or 'unexplainability' as the mark of emergence, most authors are focusing on epistemological non-derivability ${ }^{8}$.

Yet, we maintain that ontological irreducibility and epistemological non-derivability are logically distinct dimensions, and call for independent philosophical examinations. In what follows, we will discuss them separately, as two different issues.

On the one hand, we will develop throughout most of the paper a philosophical defence of emergence against the exclusion argument and the danger of epiphenomenalism, by relying exclusively on the irreducibility of emergent properties, without addressing the issue of their non-derivability. Emergent properties, we will argue, can be defined exclusively in terms of irreducibility and, crucially, they provide the system with distinctive causal powers even though they are derivable from their emergence base.

On the other hand, the issue of the non-derivability of emergents may play an important role in the discussion on whether emerging properties enable a system to exert inter-level causation between the whole and the parts. As we will suggest in the last part of the paper (section 7.2), if an emergent property is proven to be also non-derivable from the properties of the constituents, because of the epistemological gap between them, it may be possible to interpret the relation between the whole and the parts as involving inter-level causation.

\section{Irreducibility and emergence}

The aim of this section is to offer, in response to the exclusion argument, a conceptual justification of emergent properties provided with irreducible and distinctive causal powers. The core of the argument consists in suggesting that a coherent account of emergent causal powers can be obtained by rejecting the identification between the 'supervenience base' and the 'emergence base' of a property. As we will propose, a property of a whole can be functionally reducible to the set of properties of its constituents (its supervenience base) while being functionally irreducible to, and then emergent on, various categories of entities which are distinct from that set. We will argue that, once the distinction between the supervenience and emergence base is conceded, the resulting account of emergence eludes the exclusion argument and justifies the existence of distinct regimes of causation, even by maintaining the principle of the inclusivity of levels.

The argument will proceed in two steps. First, we will advocate (section 4.1) an interpretation of the relation between the whole and the parts in terms of relational mereological supervenience, according to which a supervenience relation holds between the whole and the configuration of its own constituents, and not the collection of constituents taken separately. We will then put forward a constitutive interpretation of relational supervenience, according to which supervenient properties can be in principle reduced to the configurational properties of the supervenience base. The main implication is that a supervenient property $\mathrm{M}$ and its

\footnotetext{
${ }^{8}$ Crutchfield, for instance, distinguished two different definitions and classes of models of emergence according to two different limitations in our capability "in principle” to describe emergent phenomena: nonpredictability and nondeducibility (Crutchfield, 1994).
} 
basal properties $S_{1}, \ldots S_{n}$ have identical causal powers. In the adoption of such a constitutive interpretation of relational supervenience lies the monistic stance of our perspective.

Second, we suggest (section 4.2) that, even under the constitutive and monistic interpretation of relational mereological supervenience, a relation of emergence (as irreducibility) holds not between $\mathrm{M}$ and configurational properties but, instead, between configurational properties and the properties of different categories of entities which do not belong to the configuration. As a consequence, configurations can be justifiably said to possess irreducible and emergent properties and hence be able to exert non-epiphenomenal causal powers (in particular, as recalled in section 5, as constraints).

\subsection{Supervenience and constitution}

The logic of the exclusion argument is based on the way in which the relation between an emergent property $\mathrm{M}$ of the whole $\mathrm{W}$ and the set of basal properties $N_{1}, \ldots N_{n}$ of its constituents $\mathrm{P}$ is conceived. Namely, the relation is supposed to be simultaneously one of (mereological) supervenience and functional irreducibility, while assuming at the same time, as mentioned above, the validity of the principle of inclusivity of levels.

In Mind in a Physical World, Kim paved the way for an answer to the exclusion argument able to maintain the inclusivity of levels, by clarifying the terms of the supervenience relation, and particularly specifying how the supervenience base is to be conceived. Kim argues that emergent properties are micro-based macro properties, i.e. second order properties emerging out the first-order properties and relations of the basal constituents (Kim, 1998, pp. 85-86). The central idea is that the relevant supervenience base is not a set of properties of constituents taken individually or as a collection, but rather the properties of the configuration of constituents, i.e. the whole set of inherent and relational properties of the constituents. In other words, mereological supervenience should not be interpreted as atomistic but, rather, relational (see also Thompson, 2007, pp. 427-8) ${ }^{9}$.

The move to adopt relational mereological supervenience makes configurations of constituents the relevant supervenience base. The basal properties $S_{1} \ldots S_{n}$ that bring about a supervenient property $\mathrm{M}$ are not the properties of the collection of constituents taken separately, but rather the configurational properties of the constituents qua constituents (including their mutual relations, which alter their intrinsic properties as separate elements), which appear only when the configuration is actually realised. If the basal constituents actually and collectively constitute a global pattern or system $\mathrm{W}$, then their properties would now include those generated by their being involved in specific relations and interactions with others elements.

The adoption of relational mereological supervenience has relevant implications for the question concerning the distinctive causal powers of the supervenient property with respect to

\footnotetext{
${ }^{9}$ The debate between a relational and an atomistic interpretation of the supervenience and emergence base has a long history that dates back to the first formulations of the notion of emergence in the British Emergentism. In Alexander's framework, for example, space and time, the lower level on which the whole natural world emerge, are relational concepts, not definable separately (Alexander, 1920). The opposition between atomistic and relational approaches is particularly evident in Lloyd Morgan's work. In contrast to the billiard balls model of extrinsic interactions, he presents the idea of relatedness based on inherent relations, that contribute to specify the properties of the terms involved in the relation (Lloyd Morgan, 1923, p. 19). It is also worth noting that according to some authors, Kim's reference to relations is still made in a fundamentally atomistic framework, and does not imply a clear commitment to relational mereological supervenience, which implies the idea that relations "do not simply influence the parts, but supersede or subsume their independent existence in an irreducibly relational structure” (Thompson, 2007, p. 428).
} 
its supervenience base. Indeed, the idea that emergent properties would be reducible to the properties of the constituents taken in isolation seems to be excessively committed to an atomistic view of nature, which does not take relations into account (Campbell and Bickhard, 2011). In turn, the claim that a supervenient property $M$ is in principle reducible to the set of configurational (i.e. including relations) properties $S_{1}, \ldots S_{n}$ of its constituents is more convincing (again, by assuming the principle of inclusivity of levels), since configurations are far richer and more complex determinations than the mere collection of intrinsic properties of constituents.

Accordingly, we hold that relational supervenience does not imply functional irreducibility but, on the contrary, constitution: $M$ supervenes on $S_{1}, \ldots S_{n}$ since it consists in $S_{1}, \ldots S_{n}$. A supervenient property $\mathrm{M}$ of a whole $\mathrm{W}$ corresponds to the whole set of configurational properties $S_{1}, \ldots S_{n}$ of its constituents (its supervenience base B). The set of the (relevant) configurational properties of the constituents of the system is, at least in principle, equivalent to the supervenient property. Hence, if $\mathrm{M}$ can be functionally reduced to the set $S_{1}, \ldots S_{n}$ of configurational properties of its constituents, it follows that it cannot possess distinctive causal powers ${ }^{10}$ since, in fact, they are equivalent ${ }^{11}$.

Yet, as we will claim in the following section, a coherent account of emergent properties provided with distinctive causal powers can still be provided, even under the constitutive interpretation of whole-parts relations.

\subsection{A reply to the exclusion argument}

Our reply to the exclusion argument consists in arguing that even though supervenient properties (M) have no distinctive causal powers with respect to the configurational properties $S_{1}, \ldots S_{n}$ of the constituents, $S_{1}, \ldots S_{n}$ themselves (which are equivalent to $\mathrm{M}$, because of constitution) are irreducible properties which may generate distinctive causal powers. Accordingly, $S_{1}, \ldots S_{n}$ can be said to be genuinely emergent. In other terms, there is an interpretation of emergence which is compatible with a monistic stance.

Here is our argument. A given configuration $\mathrm{C}$ of elements of a whole $\mathrm{W}$ is identified by a set of (possibly dynamic) distinctive constitutive and relational properties $S_{1}, \ldots S_{n}$. On the basis of this set of distinctive properties, a configuration is functionally irreducible to whatever entity that does not actually ${ }^{12}$ possess the same set of properties. We claim that a relation of emergence holds between a configuration $\mathrm{C}$ and any emergence base $\mathrm{P}$ whenever $\mathrm{C}$ is irreducible to $\mathrm{P}$, i.e. if $\mathrm{C}$ possesses some distinctive set of configurational properties that $\mathrm{P}$ does not possess, such that $\mathrm{C}$ does not supervene on $\mathrm{P}$. The reader would immediately note that this characterisation of emergence is very general, and could in principle include a wide range of obvious and uninteresting cases of $\mathrm{P}$, which would not be considered salient for the philosophical debate on emergence. This is correct, and we deal with this issue just below. Yet, let us point out here that, as Campbell and Bickhard (2011, p. 18; see also Teller, 1986)

\footnotetext{
${ }^{10}$ The interpretation of relational mereological supervenience in terms of constitution is consistent, we argue, with the position developed by Craver and Bechtel (2007) within their mechanistic framework. As they suggest, the relations between constituents located at different levels in a mechanism are better understood as constitutive relations (pp. 554-555). See section 7 below for a detailed discussion.

${ }^{11}$ For simplicity, we will only refer, from now on, to 'configurational properties $S_{1}, \ldots S_{n}$ ' (equivalent to 'property M'), and to 'whole W' (equivalent to 'supervenience base B' ).

12 It is important to emphasise that configurational properties must be actually realised, and not just "dispositional”. As a consequence, a configuration C is functionally irreducible, in this account, also to those entities that would possess the "potential disposition" to actualise these properties.
} 
have highlighted, appealing to configurations seems to be a sufficient answer to the danger of causal drain and epiphenomenalism. The crucial point, as we mentioned above, is that configurations include relational properties, which cannot be reduced to intrinsic properties, i.e. properties of constituents taken in isolation. Relatedness is ontological novelty. As a consequence, because of relatedness (again: actual relatedness) configurations may possess distinct causal powers that would not exist otherwise.

To avoid confusion, it is important to stress again that this account, in contrast with most existing ones, defines emergence exclusively in terms of ontological irreducibility, by leaving aside the issue of the epistemological non-derivability of $\mathrm{C}$ from $\mathrm{P}$. C is emergent on $\mathrm{P}$ if it possesses some set of new (relational) properties $S_{1}, \ldots S_{n}$ which P does not possess, and which are then irreducible to the set of property $N_{1}, \ldots N_{n}$ of P. A distinct issue, which is irrelevant here, is whether one can derive or predict $S_{1}, \ldots S_{n}$ from $N_{1}, \ldots N_{n}$. In particular, $S_{1}, \ldots S_{n}$ would be irreducible even if they were derivable, because of the novelty introduced by the relations among constituents.

At this point, given the constitutive relation between the whole and the constituents advocated so far, one may wonder what exactly configurations emerge on. Following our definition, three main kinds of emergent base $\mathrm{P}$ can be logically identified. First, the configuration $\mathrm{C}$ is not supervenient, yet is emergent on the properties of any proper subset $\mathrm{P}_{\text {sset }}$ of its constituents (its parts). A wheel has emergent properties and distinctive causal powers on any subset of itself (i.e., a half-wheel). Second, the configuration $C$ is not supervenient, yet is emergent on its substrate $\mathrm{P}_{\mathrm{sstr}}$, i.e. the collection of its constituents taken separately as if they were not constituents (so to speak, the 'potential ingredients' of a configuration). A wheel is emergent on the collection of molecules taken as if they were not actually assembled as a wheel.

Third, and importantly, the configuration $\mathrm{C}$ is not supervenient, yet is emergent on its surroundings $\mathrm{P}_{\text {surr }}$, i.e. each set of external elements that does not actually constitute $\mathrm{C}$. The wheel is emergent on each set of external molecules or entities, which are not actual constituents of it. In particular, given that a very broad set of entities might be included in $\mathrm{P}_{\text {surr, }}$ only relevant instances will actually be considered: in particular, the reference to surroundings $\mathrm{P}_{\text {surr }}$ will be restricted to those relevant $\mathrm{P}_{\text {surr }}$ on which the configuration $\mathrm{C}$ has causal effects, by virtue of its emerging properties. As we will discuss in the following section, this is precisely the relevant case with regard to biological systems.

At this point, we have all the elements required to formulate our reply to the exclusion argument. The argument claims that properties cannot be emergent unless it can be shown that they possess distinctive causal powers; at the same time, it seems that, as supervenient properties, they do not possess new causal powers with respect to their supervenience base. Hence, they are epiphenomenal. To this, we reply that emergent properties do not need to be irreducible to their supervenience base to possess distinctive causal powers: what matters is that configurations, because of relatedness, possess irreducible properties with respect to their subsets, substrate and (relevant) surroundings. Supervenience and emergence are then alternative notions: either a set of properties is supervenient on another one (in which case there is a constitutive relation between them), or it is emergent (in which case there is irreducibility).

Let us stress again that this way of conceiving emergence, interpreted exclusively as ontological irreducibility, is indeed very general. For instance, all chemical bonds are configurations emergent on their parts, substrate and surroundings, since they realise new relations, and therefore possess distinctive configurational properties. Yet, the fact that this definition covers also irrelevant or obvious cases is, we argue, the price to pay for making it compatible with the constitutive interpretation of the relations between the whole and the 
parts. More generally, we hold that this characterisation of emergence is sufficient to provide a justification for the appeal to distinctive and irreducible causal powers in the scientific discourse (Laughlin et al., 2000), and specifically in biology. Emergence appears whenever scientists are dealing with a system, as biological ones, whose properties are irreducible to those of its isolated parts, substrate and surroundings. In such cases, one must introduce new objects, relations and causal powers, which exist only within that very system, and not in its emergent base ${ }^{13}$.

\section{Emerging causal powers as constraints}

By virtue of their relatedness, configurations possess emergent properties, and may exert distinctive causal powers on their surroundings that can take different forms, following the kind of systems under consideration. Let us focus here on the case in which these causal powers are exerted as constraints, which can be organised in turn as closure.

In general terms, constraints are determinations that reduce the degrees of freedom of the system to which they belong (i.e. an inclined plane which reduces to two spatial dimensions the motion of a ball on it), simplify (or change) the description of that system, and contribute to provide an adequate explanation of its behaviour, which might otherwise be underdetermined or wrongly determined (Umerez \& Mossio, 2013).

In a given system, constraints are characterised as configurations emerging on, and acting on, specific surroundings $P_{\text {surr }}$, i.e. a set of physicochemical changes that involve the movement, alteration, consumption, and/or production of entities in conditions away from thermodynamic equilibrium. More precisely, given a particular process $\mathrm{P}_{\text {surr }}$, a configuration $\mathrm{C}$ acts as a constraint if the following conditions are fulfilled:

1) At a time scale $\tau_{\mathrm{i}}, C$ is conserved throughout $P_{\text {surr }}$, i.e. there is a set of emerging properties $S_{1}, \ldots S_{n}$ of $\mathrm{C}$ which remain unaffected throughout $\mathrm{P}_{\text {surr }}$;

2) At $\tau_{\mathrm{i}}, C$ exerts a causal role on $P_{\text {surr }}$, i.e. there is some observable difference between $\mathrm{P}_{\text {surr }}$ and $\mathrm{P}_{\text {surr }}{ }^{\mathrm{c}}\left(\mathrm{P}_{\text {surr }}{ }^{\mathrm{c}}\right.$ is $\mathrm{P}_{\text {surr }}$ under the causal influence of $\mathrm{C}$ by virtue of properties $\left.S_{1}, \ldots S_{n}\right)$.

It is worth noting that, usually, $\mathrm{C}$ does not extend the set of possible behaviours of $\mathrm{P}_{\text {surr, }}$ i.e. $\mathrm{P}_{\text {surr }}$ could in principle (although very unlikely) exhibit, at different time scales, the behaviour of $\mathrm{P}_{\text {surr }}{ }^{\mathrm{C}}$ without the action of $\mathrm{C}$.

Consider the example of the vascular system, taken as a global constraint (C) on the flow of oxygen $\left(\mathrm{P}_{\text {surr }}\right)$ in an organism. First, the topology of the vascular system remains unaltered during the oxygen flow (at least at relatively short scales $\tau_{\mathrm{i}}$ ). Second, the vascular system exerts a causal role on the flow of oxygen, since, for instance, the flow takes the form of a transport (canalised) to the neighbourhood of each cell, instead of having a diffusive (unconstrained) form. In this case, moreover, the flow of oxygen could reach (at least in principle, since in practice this would be extremely unlikely) each cell at an adequate rate even in the absence of the vascular system, from the point of view of statistical mechanics.

For the purposes of this paper, two features of the concept of constraint have to be emphasised.

\footnotetext{
${ }^{13}$ It is worth noting that the relation between the emergent properties and its emergence base can be interpreted both synchronically and diachronically. Being based on novelty, in fact, the irreducibility to any entity that does not belong to an actual configuration is in principle compatible with both the dimensions of emergence. See also footnote 21 below.
} 
First, insofar as they are causal effects produced by emerging properties of configurations, constraints depend on the existence of the relevant configuration. Hence, constraints are not universal, but rather local and contingent causes. In particular, configurations fit the definition only at some specific time scales, whereas at different scales they usually fail to satisfy one or more conditions. For instance, at time scales larger than $\tau_{\mathrm{i}}$, configurations are normally subject to degradation, and must be replaced or repaired (and then fail fitting condition 2). Yet, at $\tau_{\mathrm{i}}$, constraints are irreducible to the thermodynamic flow (in particular because of their conservation) and require an alternative description (Pattee, 1972). Because of their distinctive causal powers, which cannot be reduced to those of their emergent base $\mathrm{P}$, constraints constitute an emergent regime of causation, operating in addition to physicochemical changes.

Second, in the description of physical systems, constraints are in most cases introduced as external determinations (boundary conditions, parameters...), such that they exert some causal influence on $\mathrm{P}_{\text {surr }}$, while their existence (or, more precisely: the maintenance of the specific properties $S_{1}, \ldots S_{n}$ providing them with relevant causal powers) does not depend on $\mathrm{P}_{\text {surr }}$. For instance, the inclined plane constrains the dynamics of the ball, but the constrained dynamics do not have a causal role on the existence or maintenance of the plane.

The appeal to constraints to provide adequate descriptions and explanations is ubiquitous in natural sciences. In the biological domain, however, constraints play a characteristic role to the extent that they give rise to a specific causal regime of self-maintenance, that we label closure.

\section{Self-maintenance: from self-organisation to closure}

In many natural systems, the relation between a constraint $C$ and its emergent base $P_{\text {surr }}$ is oriented, in the sense that $C$ may causally act on $\mathrm{P}_{\text {surr }}$, but not vice-versa. Yet, there are cases in which $\mathrm{C}$ acts on $\mathrm{P}_{\text {surr }}$ that, once constrained, contributes to determine (at least some of) the boundary conditions at which $\mathrm{C}$ exists. To use our labels, there are cases in which $\mathrm{C}$ constrains $\mathrm{P}_{\text {surr }}$ such that the constrained $\mathrm{P}_{\text {surr }}{ }^{\mathrm{c}}$ constitute some of the boundary conditions required for $C$ to exist. In that case, the resulting system realises self-maintenance, since $C$ constrains $\mathrm{P}_{\text {surr }}$, which in turn, once constrained, maintains $\mathrm{C}$. If $\mathrm{C}$ did not act on $\mathrm{P}_{\text {surr, }}$, it would not (or it would cease to) exist. Self-maintaining systems are then systems able to maintain some of the conditions required for their own existence, by virtue of the constraining action of their own configurations (Mossio \& Moreno, 2010, p. 272; see also Kauffman, 2000, for a related analysis in terms of 'work-constraint cycles').

Self-maintenance exists in the physical and chemical domain. The classical example are dissipative structures (Glansdorff \& Prigogine, 1971; Nicolis \& Prigogine, 1977), in which a huge number of microscopic elements adopt a global, macroscopic ordered configuration (a 'structure') in the presence of a specific flow of energy and matter in far-fromthermodynamic equilibrium (FFE) conditions. In turn, the macroscopic configuration exerts a constraint that contributes to the maintenance of the FFE flow of energy and matter enabling the persistence of the microscopic dynamics (Ruiz-Mirazo, 2001, p. 59).

A number of physical and chemical systems, such as Bénard cells, flames, hurricanes, and oscillatory chemical reactions, can be pertinently described as self-maintaining dissipative systems. Let's take the example of 'Bénard cells', i.e. macroscopic structures that appear spontaneously in a liquid when heat is applied from below (Chandrasekhar, 1961). In the initial situation, in which there is no difference in temperature between upper and lower layers, the liquid appears uniform, in terms of the statistical distribution of the kinetic energy 
of the molecules. When heat is applied, and the temperature in the lower layer is increased up to a specific threshold, the liquid's dynamics change dramatically: the random movements of the microscopic molecules spontaneously become ordered, creating a macroscopic pattern (convection cells). In each cell, billions of microscopic molecules rotate in a coherent manner along a hexagonal path, either clockwise or anticlockwise, and always in the opposite direction from that of its immediate neighbours in a horizontal plane.

Bénard cells appear when $\mathrm{P}_{\text {surr }}$ realises some specific boundary conditions (e.g. the heat applied from below), which exert external constraints on the dynamics. Yet, once they have appeared, the maintenance of Bénard cells depends not only on those independent boundary conditions, but also on some constraint exerted by the very configuration on its surroundings. For instance, the cells capture surrounding water molecules in their dynamics, turning them into constituents. It is through this action that Bénard cells contribute to maintain some of the boundary condition at which the flow of energy and matter traversing them may occur. With our labels, the emerging configuration $\mathrm{C}$ acts as a constraint on $\mathrm{P}_{\text {surr, }}$ on which it emerges, by turning them into appropriate boundary conditions $\mathrm{P}_{\text {surr }}{ }^{\mathrm{c}}$. We need then to appeal to $\mathrm{C}$ itself to explain its own maintenance, which would otherwise be impossible (or at least very unlikely) on the basis of the unconstrained properties of $\mathrm{P}_{\text {surr }}$.

As it has been recently emphasised (Mossio \& Moreno, 2010), dissipative systems realise a minimal form of self-maintenance, in the sense that they generate a single macroscopic structure acting as a constraint on its surroundings. Accordingly, dissipative systems make a unique contribution to their own maintenance, since they contribute to maintain the unique constraint involved in the self-maintaining loop between $\mathrm{C}$ and $\mathrm{P}_{\text {surr }}$.

When considering biological systems, the situation is more complex. Unlike minimal selfmaintaining systems, biological systems generate a network of structures, exerting mutual constraining actions on their boundary conditions, such that the whole organisation of constraints realises collective self-maintenance. In biological systems, constraints are not able to achieve self-maintenance individually or locally: each of them exists insofar as it contributes to maintain the whole organisation of constraints that, in turn, maintains (at least some of) its own boundary conditions. Such mutual dependence between a set of constraints is what we call closure, the causal regime that, we claim, is paradigmatically at work in biological systems ${ }^{14}$.

Let's give a more formal characterisation. A system realises closure if and only if it contains a set of structures $C_{1} \ldots C_{n}$ acting as constraints at the relevant time scales $\tau_{1} \ldots \tau_{n}$, by virtue of emerging properties $S_{1}, \ldots S_{n}$ such that, for each constraint $\mathrm{C}_{\mathrm{i}}$, (at least some of) the boundary conditions required for the maintenance of the relevant emergent property $\mathrm{M}_{\mathrm{i}}$ are determined by the causal action of another constraint $C_{j}$ by virtue of a property $\mathrm{M}_{\mathrm{j}}$, whose maintenance depends in turn on the action of $\mathrm{C}_{\mathrm{i}}$.

As all dissipative systems, be they physical or chemical, biological systems are traversed by a flow of energy and matter, which takes the form of processes and reactions occurring in open thermodynamic conditions. What specifically characterises biological systems ${ }^{15}$ is the fact that the thermodynamic flow is constrained and canalised by a set of constraints in such a way that they realise a mutual dependence. In this sense, biological organisation is a specific kind

\footnotetext{
14 The concept of closure has been proposed by several influential authors, that we have mentioned in the introduction. For a recent survey, see Chandler \& Van De Vijver (2000). Mossio (2013) provides a synthetic overview of the meaning and uses of the term in the biological domain.

${ }^{15}$ Or, at least, systems being 'at the edge' of the biological domain. We do not discuss this issue here, since it does not interfere with the central point.
} 
of higher-level configuration, which achieves self-determination as collective selfconstriction: the conditions of existence of (at least a subset of) the constitutive constraints are, because of closure, mutually determined within the very organisation. In what follows, the term 'organisation' will then specifically refer to this kind of higher-level configurations, whose constituents are themselves configurations, each of them acting as constraints.

This characterisation is, of course, very general and lacks several specifications about the way in which closure is actually realised in biological systems. Yet, it is precise enough for the purposes of this paper, and in particular, allows discussing three crucial implications.

First, the closed regime of dependence among constraints is emergent on, and irreducible to, the open regime of thermodynamic processes and changes. Closure is realised through the mutual causal action of the constraints which, as discussed in section 5, are irreducible to the thermodynamic flow, each constraint being conserved at the relevant time scale $\tau_{1} \ldots \tau_{\mathrm{n}}$. As a consequence, a reductive description of closure in terms of the causal regime of thermodynamic changes would be inadequate, since it would be unable to include constraints and their contribution as causal factors ${ }^{16}$. In particular, a description of biological organisation not appealing to the causal power of constraints and their closure would amount to a system constituted by a cluster of unconnected processes and reactions, whose coordinated occurrence could be theoretically possible at very large time scales (see the definition of constraints above), but extremely unlikely - and often impossible - at biologically relevant time scales ${ }^{17}$.

Second, organisations themselves possess, because of closure, emergent properties. One of them, which is particularly relevant here, is the generation of functions. As it has been recently argued (Mossio et al., 2009; Saborido et al., 2011), when subject to closure, constraints correspond to biological functions: performing a function, in this view, is equivalent to exerting a constraining action on an underlying process or reaction ${ }^{18}$. All kinds of biological structures and traits to which functions can be ascribed satisfy the above definition of constraint, although at very different temporal and spatial scales. Some intuitive examples, in addition to the vascular system mentioned above, include, at different scales: enzymes (which constrain reactions), membrane pumps and channels (which constrain the flow of ions through the membrane), organs (as the heart which constrains the flow of blood), and so on. The emergence of closure is then the emergence of functionality within biological organisation: constraints do not exert functions when taken in isolation, but only insofar as they are subject to a closed emergent organisation.

Third, closure is specifically defined with respect to the emergence base $\mathrm{P}_{\text {surr }}$ constituted by a set of processes and changes occurring in conditions far from thermodynamic equilibrium.

16 It is of course conceivable that a description of constraints might possibly be given in terms of thermodynamics, specifically as entities not being affected by the thermodynamic flow. However, in this case, constraints (and then closure) would not be reduced to a different causal regime, but simply redescribed in different terms.

17 This allows distinguishing, moreover, a closure of constraints from a cycle of processes or reactions as, for instance, the water cycle. In the case of cycles, the entities and processes involved in the cycle (e.g. clouds, rain, spring, river, sea, clouds...) do not act as constraints on each other, and the system can be adequately described by appealing to a set of external boundary conditions (ground, sun...) acting on a single causal regime of thermodynamic changes (see also Mossio \& Moreno, 2010).

${ }^{18}$ In this framework, functions are interpreted in an organisational sense: a trait is functional if and only if it exerts a constraint that is subject to closure and, consequently, contributes to the maintenance of the organisation while being maintained by that very organisation. As extensively discussed in Mossio et al. (2009) the organisational account of functions integrates in a unified framework both etiological and causal-systemic theories of function. 
Although mutually irreducible, the two causal regimes realise a two-way interaction in biological systems, to the extent that constraints act on thermodynamic processes and changes, which in turn contribute to reproducing or maintaining these constraints. Hence, it might be tempting to conclude that closure (just as any form of self-maintenance) inherently involves not just emergent causation but also inter-level causation, at work between the two causal regimes. Yet, there are several reasons to resist the temptation, at least insofar as particularly controversial kinds of inter-level causation are invoked.

\section{Inter-level causation}

The issue of inter-level (be it upward or downward) causation has been, more or less explicitly, a central aspect of the debate about emergence since its beginnings ${ }^{19}$, since the very concept of emergence carries on the issue of the relations between properties at different levels.

In Kim's account, attributing causal powers to emergent properties necessarily implies downward causation. Let's recall his argument that, as we discussed, identifies the supervenience and the emergence bases. Let be $\mathrm{M}$ and $\mathrm{M}^{*}$ two emerging properties, and suppose that $\mathbf{M}$ causes $\mathrm{M}^{*}$ (a case of 'same-level' causation). As an emergent property, $\mathbf{M}^{*}$ has an emergence base, say $\mathrm{P}^{*}$. Given the supervenience relation, $\mathrm{P}^{*}$ is necessary and sufficient for $\mathrm{M}^{*}$ : if $\mathrm{P}^{*}$ is present at a given time, then $\mathrm{M}^{*}$ is also present. Accordingly, it is unclear in what sense $\mathrm{M}$ could play a causal role in bringing about $\mathrm{M}^{*}$ : given $\mathrm{P}^{*}$, its role would be useless, unless $\mathrm{M}$ has in fact something to do in causing $\mathrm{P}^{*}$. In other words, the same-level causation of an emergent property makes sense only if this implies the causation of the "appropriate basal conditions from which it will emerge" (Kim, 2006, p. 558). As a consequence, causation produced by emergent properties seems to imply, in all cases, downward causation in the sense of a causal influence exerted by an emergent property on the basal conditions of another emergent property.

Yet, as Kim himself has argued (Kim, 2010), this general form of downward causation, i.e. a causal influence exerted by an entity at a higher-level on a distinct entity located at a lower level, is indeed widespread and unproblematic. In particular, this interpretation of downward causation applies straightforwardly to self-maintenance and closure, which inherently involve, as discussed, the upward and downward causation between constraints and dynamics, each of them located at different levels of description (see also section 7.2 below).

The more controversial form of downward causation would be that exerted by a whole on its own constituents (in Kim's terms, "reflexive” downward causation, 2010, p. 33). According to Emmeche et al. (2000), there are various possible interpretations of reflexive upward and downward causation. In their view, the only non-contradictory versions of the concept are those interpreting downward causation in terms of 'formal' causation (2000, pp. 31-32), such that the whole exerts a constraining action on its own constituents, by selecting specific behaviours among a set of possible ones. This interpretation can be taken, as Emmeche and

\footnotetext{
${ }^{19}$ According to Lloyd Morgan, “[...] when some new kind of relatedness is supervenient (say at the level of life), the way in which the physical events which are involved run their course is different in virtue of its presencedifferent from what it would have been if life had been absent. [...]. I shall say that this new manner in which lower events happen - this touch of novelty in evolutionary advance depends on the new kind of relatedness" (Lloyd Morgan, 1923, p. 16). According to Stephan (1992) Lloyd Morgan’s passage could admit different interpretations, such as that of a logical claim about supervenience. On the contrary, McLaughlin asserts: "In Morgan one finds the notion of downward causation clearly and forcefully articulated” (McLaughlin, 1992, p. 68).
} 
co-authors claim, as the standard and possibly more compelling one of downward causation, and it is very close to the original proposal by Campbell $(1974)^{20}$.

As an illustration, consider Sperry's classical example of the wheel rolling downhill (Sperry, 1969). On the one side, the various molecules generate the wheel as a whole. On the other side, as Emmeche et al. (2000, p. 24) explain, "none of the single molecules constituting the wheel or gravity's pull on them are sufficient to explain the rolling movement. To explain this one must recur to the higher level at which the form of the wheel becomes conceivable". The set of configurational properties of molecules is supposed here to underdetermine their behaviour so that, in order to explain it, one needs to appeal to a property of the whole (in this case: the form of the wheel) that would generate a causal influence (a selective constraint) exerted on its own constituents.

Because of the (supposed) under-determination of constituents by configurational (intrinsic and relational) properties, constituents' behaviour is partly determined, in a functionally irreducible way, by the whole to which they belong. In particular, this train of thought seems to equally apply to biological systems, in which the behaviour and dynamics of the parts appears to be, in an important sense, determined (notably through regulation and control functions) by the downward causation exerted by the whole system to which they belong.

In what follows, we will examine whether self-maintenance and closure do involve some form of reflexive inter-level causation, intended as a particular form of constraint exerted by the whole on its parts. As we will argue (7.1), there seems to be no compelling argument in favour of a positive answer in our framework, at least under the monistic assumptions adopted so far. Alternative conclusions could be obtained (7.2) by rejecting some of these assumptions, or by shifting the analysis to an epistemological or heuristic dimension.

Before continuing, a terminological clarification. A possible objection might contend that this debate somehow forces a narrow understanding of inter-level causation in terms of reflexive whole-parts causal influence, whereas the usual meaning in the biological domain refers to the non-reflexive case, where higher-level entities interact with lower-level entities, the latter not being constituents of the former. Indeed, this interpretation of inter-level causation applies straightforwardly to biological organisation, and is inherently involved in the very notion of closure. In this sense, biological discourse requires a general concept of inter-level causation. To avoid ambiguities, we propose using different terms to refer to the two ideas: in what follows "inter-level causation" will be therefore used for the general non-reflexive case, and "nested causation" for the reflexive whole-parts case.

This way, biological descriptions would be able to refer to inter-level causation, while at the same time avoiding incongruities with philosophical analyses.

\subsection{Why we do not need nested inter-level causation in biology}

The account of emergence and supervenience developed so far has relevant implications on the conception of nested causation ${ }^{21}$.

\footnotetext{
${ }^{20}$ Campbell defines downward causation as follows: "all processes at the lower level of a hierarchy are restrained by and act in conformity to the laws of the higher level” (1974, p. 180).

${ }^{21}$ In the philosophical literature, nested causation comes in two variants, synchronic and diachronic (Kim, 2010, pp. 34-36). On the one hand, synchronic nested causation refers to the situation in which upward and downward causation would occur simultaneously. In more technical terms, a supervenient property $\mathrm{M}$ acts causally on its supervenience base $S_{1} \ldots S_{n}$ at the same time that the supervenience base generates $\mathrm{M}$. On the other hand, diachronic (or diagonal) nested causation refers to the situation in which $\mathrm{M}$ acts on its own supervenience base $S_{1}, \ldots S_{n}$, causing its modification, but only at a subsequent time with respect to the upward determination. In this
} 
Concerning the supervenience base B - insofar as the principle of inclusivity of levels is maintained (but see section 7.2 below), and the relation between an emergent property $\mathrm{M}$ and the configurational properties $S_{1}, \ldots S_{n}$ of $\mathrm{B}$ is conceived as constitutive - the exclusion argument applies more cogently to relational supervenience than to its atomistic version. As a consequence, as Craver and Bechtel (2007) emphasise, no nested causation can exist between an emergent property and its own supervenience base: there is no justification for claiming either that $S_{1}, \ldots S_{n}$ 'generate' or 'produce' $M$, or that M exerts downward causation on $S_{1}, \ldots S_{n}$. In particular, the closed organisation does not exert causation on the whole network of constitutive constraints, and the whole network of constitutive constraints does not produce the closed organisation. Under the monistic stance adopted so far, there is therefore no room for nested causation.

Let us consider now the emergence base $\mathrm{P}$ of $\mathrm{C}$, and its different versions discussed in section 4. Is there nested causation between the configuration $\mathrm{C}$ and any subset $\mathrm{P}_{\text {sset }}$ of its constituents? In our view, by assuming the principle of the inclusivity of levels, the answer is no, since the properties of each $\mathrm{P}_{\text {sset }}$ (which may refer, for instance, to each individual constraint) are by definition configurational, so that the appeal to some constraint exerted by the whole would be redundant: configurational properties are such precisely because an entity belongs to a whole. Also, no nested causation occurs between the whole and its substrate $\mathrm{P}_{\text {sstr }}$ because, in our account, the collection of its constituents taken separately (without their configurational properties) is an abstract description that does not correspond to the way in which constituents are organised in the system. Since, in the system there is no such a thing as the collection of unrelated constituents, they cannot, a fortiori, be involved in nested causation, or indeed any causation at all.

The case of the third kind of emergence base, the surroundings $\mathrm{P}_{\text {surr }}$, is somehow different. As we discussed in sections 5 and 6, emergent configurations do exert a causal action on their surroundings, notably in the form of constraints. Yet, surroundings are by definition external to the configuration, which means that the constraints exerted by $\mathrm{C}$ on $\mathrm{P}_{\text {surr }}$ can by no means be interpreted in terms of nested causation.

The claim according to which constraints, in our framework, do exert causal powers, but not in the form of nested causation, has crucial consequences on the interpretation of selfmaintenance and closure.

In the case of physical self-maintaining systems, the fact that the emergent configuration acts to maintain itself does not appear to constitute, per se, a case of nested causation, since the constraining action is exerted on the surroundings of the configuration, not on its own constituents. Let us consider again the example of Bénard cells. An interpretation appealing to nested causation would claim that each cell (i.e. the emergent configuration) exerts a constraint on its own microscopic constituents, in the sense that the fact of belonging to a given cell determines whether a molecule rotates in a clockwise or counter-clockwise direction. As Juarrero (2009, p. 85) puts this: "Once each water molecule is captured in the dynamics of a rolling hexagonal Bénard cell it is no longer related to the other molecules just externally; its behaviour is contextually constrained by the global structure which it constitutes and into which it is caught up. That is, its behaviour is what it is in virtue of the individual water molecules' participation in a global structure”.

Yet, what we call the cell is the configuration of constituents, so that, as we argued above, it is redundant to appeal to the whole set of constituents and relations to explain the behaviour

paper, however, we assume that the distinction is irrelevant, since we question the very idea of the causal influence of $\mathrm{M}$ on $S_{1}, \ldots S_{n}$, be it synchronic or diachronic. 
of each constituent, whose characterisation already includes its relational properties as part of the configuration. Once a given molecule has been 'captured' by the cell and has begun to rotate with the others, in what sense would it still be "constrained by the global structure"22?

Two reasons may explain why self-maintaining systems seem to be a case of nested causation. First, the description of the configurational properties of dissipative structures, which is available at a given moment, usually under-determines their behaviour. This is of course a crucial point; still, as we discussed earlier, this should not be taken as a sufficient reason to appeal to nested causal relations since, as we pointed out in section 3, it confuses epistemological non-derivability with ontological irreducibility (but see section 7.2. below). Second, self-maintaining systems would not exist if they did not generate a causal loop between the whole configuration and its constituents. Yet, the crucial point is that, in our view, this loop is not a direct loop, but rather an indirect one, realised through the action of the constraint on its surroundings. What might appear as an action exerted on the constituents is in fact exerted on the boundary conditions of these constituents.

In the light of these considerations, in particular, we do not think that the appeal to the supposed constraint exerted by the configuration on its own constituents in terms of formal causation is explanatory (again, under the monist assumptions adopted so far). The formal causation of the whole on its constituents would be in principle reducible to the constraining action exerted on the boundary conditions of these constituents, without loss of information or explanatory power.

Let us now examine closure. Is there a characteristic aspect of closure that would justify, in contrast to simple self-maintenance, the claim according to which it realises nested causation?

The main difference between physical self-maintenance and closure is that, in the second case, self-maintenance is realised collectively, by a network of mutually dependent constraints. In real biological systems, closure is realised though a very complex organisation of constraints, such that, in most cases, a given constraint exerts its action on surroundings that have already been subject to the causal influence of at least one other constraint. For instance, most enzymes act on reactions whose reactants are the result of the joint action of other constraints, including the membrane (through its channels and pumps). In these cases, it can be said that constraints act on entities which are already 'within' the system, at least in the sense of having already been constrained by the system. This seems to be a clear difference with respect to simple self-maintaining systems, and one may then conclude that the closed organisation does act on its own constituents, and realises nested causation.

Yet, we hold that the conclusion is incorrect, since it interprets those constrained processes and reactions as constituents of the organisation (which, we recall, is a higher-level configuration of constraints), whereas they are not. In biological systems, the constituents of the organisation are the constraints themselves, which realise collective self-maintenance. Under the constitutive interpretation of the relation between the whole and its constituents, the organisation as such does not possess emergent and distinctive causal powers with respect to the network of constraints which, in turn, exerts causal powers on surroundings which are not themselves constituents of the network (although they usually are within the spatial

\footnotetext{
${ }^{22}$ A satisfactory analysis of downward causation, then, requires a careful distinction between two ideas. One is the idea that a configuration is made up by a set of constituents, which have causal interactions between them. Explaining why a given molecule of water is rotating in a given manner at a given moment requires an appeal to its causal interactions with other constituents. And the reason why a set of constituents may exert a causal influence on other constituents is, of course, that all of them belong to the same system. The other idea, in contrast, is that the 'whole system', including any specific constituent, would have a causal role on that very constituent.
} 
borders of the system) $)^{23}$. Accordingly, we maintain that closure does involve inter-level causation, but not nested causation.

A second reason why closure seems to inherently imply nested causation is that evoked by Kant (Kant, [1781] 1987), i.e. the fact that the existence of the constituents (the constraints) 'depends on the whole'. Indeed, the mutual dependence among constraints is a fundamental difference between organisations and other configurations. In the second case the existence and maintenance of the constituents does not depend on their being involved in the configuration: one can decompose a wheel into its molecular elements, which would continue to exist as separate elements. The same holds for the microscopic constituents of a dissipative system. In contrast, closed organisations imply a more 'existential' kind of relation among constituents (the constraints themselves), which exist as far as they are involved in the whole organisation. Actually, the appeal to formal causation advocated by several authors is essentially aimed, in our view, at capturing this distinctive feature of biological organisms.

Yet, these specific features of closed organisations do not require ascribing distinctive causal powers to the whole, since closure can be realised through the network of mutual, usually hierarchical, causal interactions. 'Depending on the whole', therefore, could simply mean 'depending on the whole network of interactions' without appealing to the whole as causal agent emergent on its own supervenience base. This interpretation of the whole-parts relation in biological organisation is particularly relevant because it applies to all those cases in which biological literature typically appeals to nested causation, i.e. all kinds of regulation and control mechanisms, - very common in biological systems - thanks to which organisms are able to (adaptively) compensate for internal and/or external perturbations (Piaget, 1967; Fell, 1997). What is frequently described as a causal action of the whole system on its own constituents, is in fact the result of the interaction among hierarchically organised constraints (or subsystems of constraints) which can result, for instance, in the acceleration of the heart rate and glucose metabolism when the organism starts playing tennis (see Craver and Bechtel, 2007, p. 559, for a detailed description of this example, and other relevant ones). Regulation and control can be understood in terms of (non-nested) inter-level causal interactions among constraints: although they inherently require, as all biological functions do, the realisation of closure as an emergent causal regime (see section 6 above), they do not involve nested causation exerted by the whole organism.

\subsection{Why we might need, after all, nested causation in biology}

The rejection of nested causation depends on the constitutive interpretation of the supervenience relation adopted so far. Indeed, the central goal of the analysis was to suggest that closure can be justifiably taken as an emergent and distinctively biological regime of causation even under a constitutive interpretation of supervenience. Yet, several strategies could be adopted to justify nested causation, and they might be successful and operational in some cases, including the biological domain, which is specifically under study here. To date, however, we think that these strategies lack any compelling argument in favour of their adoption in the biological domain; their relevance is still under conceptual and scientific scrutiny. That is why, in our view, the constitutive interpretation of the whole-parts relation is still the wiser one. Let us discuss these strategies.

23 The physical processes on which the network exerts (constraining) causal powers can, in some cases, become members of the network itself, when they enter into configurations which act as constraints. Nonetheless, the network would exert causal powers on them as long as they are part of its surroundings, and it would cease acting causally on them as soon as they would start playing the role of constraints. 
The first strategy is ontological and advocates that a non-constitutive interpretation of relational supervenience should be adopted, in order to admit causation of the whole on the constituents. In this interpretation, emergent properties can be at the same time supervenient on and irreducible to configurations. For this ontological stance to be coherent, one must accept the violation of the inclusivity of levels, hence accepting the idea that the very same entity (say: a constituent of a configuration) may possess different properties, and therefore obey different laws or principles, at different levels of description. In other terms, it consists in rejecting the monistic stance advocated so far. For instance, each molecule constituting the wheel would have the property of behaving in a given way when considering the whole configuration, but each of them would not possess the same property when looked at individually. Even though we are looking at the very same molecules under the very same conditions, their properties would vary according to the level of description, since the relevant laws and principle would vary.

In our view, rejecting the principle of the inclusivity of levels could be indeed an important tool to adequately account for natural phenomena that would require appealing to nested causation. We have no principled objections to this position. Yet, we maintain that its relevance for the biological domain is still uncertain. As Craver \& Bechtel (2007) have convincingly argued, many (or most) apparent biological examples of downward causation (in particular cases of 'downward' regulation) seem to be adequately explainable by hybrid accounts appealing to intra-level causal interactions between constituents and inter-level constitutive relations. In those cases, an advocate of the constitutive interpretation of mereological supervenience could argue that the appeal to nested causation seems precisely to stem from an inadequate understanding of the role of configurations: the behaviour of the constituents appears to be influenced by the whole because the description focuses only on the internal properties of the constituents, neglecting the relational ones ${ }^{24}$. In a word, there seems to be no clear case in the biological domain for which the appeal to nested causation is mandatory. Self-maintenance and closure are no exceptions in this respect.

The second strategy is epistemological, and consists in justifying nested causation by demonstrating that it would be impossible, in principle, to determine the behaviour of a system through a description of its configurational properties. On the basis of such negative result, the appeal to nested causation would be justified in epistemological terms, since there would be, in principle, no alternative description ${ }^{25}$. Yet, while arguments of 'inaccessibility' have already been formulated in physics (Silberstein \& McGeever, 1999), this is not the case

\footnotetext{
${ }^{24}$ In the case of the wheel, for instance, one may say that if we describe a given molecule as a constituent of a wheel, we are already including in the description all constitutive and relational properties, which make it a constituent ('being in such and such position', 'having such and such interactions and links with neighbouring molecules'...), and which determine its behaviour under specific conditions. For instance, a force (i.e. gravity) applied to a part will generate a chain of causal interactions among the constituents which, because of their individual configurational properties, will behave in a specific way. We will then call the collective pattern the 'rolling movement of the wheel'. Each molecule of the wheel will move in a specific way because its configurational properties force it to do so, and a complete description of the configurational properties of the individual constituent will suffice to explain why it behaves as it does. The fact that the constituents collectively constitute a wheel, whose macroscopic behaviour can be described as a rolling movement, does not add anything to the explanation of the individual behaviour. There are indeed causal interactions here, but not inter-level causation.

${ }^{25}$ See Bich (2012) for an epistemological discussion of the relationship between emergence and downward causation.
} 
in biology ${ }^{26}$. As a consequence, there seems to be, to date, no compelling epistemological arguments imposing to admit nested causation for biological systems.

The third strategy is heuristic. There are in fact many cases, especially in complex systems, in which the available description of the configurational properties is insufficient to adequately determine the behaviour of the whole system. In those cases, which are indeed widespread, it might be useful to appeal to the configuration as a whole as if, by virtue of its emergent properties, it were exerting nested causation on its constituents, so to provide a description capable of sufficiently determining the behaviour of the system. Since it is not committed to a theoretical non-constitutive interpretation of supervenience, the heuristic appeal to nested causation can be adopted as a pragmatic tool even within a constitutive interpretation of supervenience. Yet, such a heuristic use of nested causation would not point to specific features of the causal regime at work in biology (which is the object of this paper), but it would simply correspond to a scientific practice common to several scientific domains. In particular, as we mentioned above, the strategy can be adopted for self-maintaining and closed systems for which, mostly because of their internal complexity, complete descriptions of their organisation are difficult to elaborate.

\section{Conclusions}

The main claim of this paper is that the organisation of biological systems can be shown to realise a distinctive causal regime, that we labelled closure. Closure translates into contemporary terms the original Kantian idea, according to which living systems can be conceived as natural purposes, in which each part exists with respect to the other parts, such that the whole is able to self-maintain. In order for closure to be a legitimate scientific concept - and not just an epistemic shortcut - philosophical arguments must be provided in favour of its emergent and irreducible character with respect to the causal regimes at work in other classes of natural systems. To attain this objective, we developed a twofold argument.

On the one hand, we argued that closure is to be conceived as the mutual dependence among a set of constituents, each of them acting as a constraint. Constraints are configurations which, by virtue of the relations among their own constituents, possess emergent properties enabling them to exert distinctive causal powers on their surroundings, and specifically on thermodynamic processes and reactions. When a set of constraints realises closure, the resulting organisation constitutes a higher-level emergent regime of causation, possessing irreducible properties and causal powers. In particular, closed organisations are able to selfmaintain as a whole (whereas none of the constitutive constraints can do it) which, in turn, enables them to generate biological functions.

On the other hand, we advocated the idea that a coherent defence of closure as an emergent and irreducible causal regime does not need to invoke nested causation. Closed organisations can be understood in terms of causal interactions among mutually dependent (sets of) constraints, without implying upward or downward causal actions between the whole and the parts. Biological emergence, accordingly, is logically distinct from nested causation, and one can advocate the former without being committed with the latter.

Again we by no means want to exclude the possibility that biological organisation might involve nested causation. As we discussed, various strategies could be adopted to adequately

\footnotetext{
${ }^{26}$ It should be noted, however, that the issue is currently being explored by several biologists and theoreticians. For instance, a relevant proposal in this direction has recently been developed by Soto, Sonnenschein and Miquel (Soto et al., 2008).
} 
justify this idea, and promising explorations are currently underway. Nevertheless, we believe that these attempts are, as yet, incomplete, and do not offer compelling arguments in the biological domain. That is why we argued that biological organisation can be shown to be emergent and irreducible even though nested causation is, by hypothesis, ruled out.

\section{Acknowledgments}

The authors wish to thank Jon Umerez and Agustin Vicente for very valuable feedback on earlier versions of this paper. This work was funded by Ministerio de Ciencia y Innovación, Spain ('Juan de la Cierva' program to LB); Research Projects of the Spanish Government (FFU2009-12895-CO2-02 to AM and FFI2011-25665 to AM and LB); Research Projects of the Basque Government (IT 505-10 and IT 590-13to AM and LB).

\section{References}

Alexander, S. (1920). Space, Time and Deity. London: Macmillan

Bich, L. (2012). Complex emergence and the living organization: An epistemological framework for biology. Synthese, 185, 215-232

Bitbol, M. (2007). Ontology, Matter and Emergence. Phenomenology and the Cognitive Science,6, 293-307

Block, N. (2003). Do Causal Powers Drain Away? Philosophy and Phenomenological Research, 67(1), 133-150

Broad, C. D. (1925). The Mind and its Place in Nature. London: Routledge and Kegan Paul Ltd

Campbell, D. T. (1974). Downward Causation in Hierarchically Organized Biological Systems. In F. J. Ayala \& T. Dobzhansky (Eds.), Studies in the Philosophy of Biology (pp. 179-186). Berkeley and Los Angeles: University of California Press.

Campbell, R. J., \& Bickhard, M. H. (2011). Physicalism, Emergence and Downward Causation. Axiomathes, 21(1), 33-56. Quotations from the online version: http://www.lehigh.edu/ mhb0/physicalemergence.pdf

Chandler, J. L. R., \& Van De Vijver, G. (Eds.) (2000). Closure: Emergent Organizations and Their Dynamics. Annals of the New York Academy of Science, vol. 901

Chandrasekhar, S: (1961). Hydrodynamic and Hydromagnetic Stability. Oxford: Clarendon Press

Cornish-Bowden, A., Cárdenas M. L., Letelier J.-C., \& Soto Andrade, J. (2007). Beyond Reductionism: metabolic circularity as a guiding vision for a real biology of systems. Proteomics, 7, 839-845

Craver, C. F., \& Bechtel, W. (2007). Top-down causation without top-down causes. Biology and Philosophy, 22, 547-563

Crutchfield, J. P. (1994). The Calculi of Emergence: Computations, Dynamics, and Induction. Physica D, 75, 11-54

Emmeche, C., Køppe, S., \& Stjernfelt, F. (2000). Levels, Emergence, and Three Versions of Downward Causation. In P. B. Andersen, C. Emmeche, N. O. Finnemann, \& P. V. Christensen (Eds.), Downward Causation (pp. 13-34). Aarhus: Aarhus University Press.

Fell, D. (1997). Understanding the Control of Metabolism. London: Portland University Press

Ganti, T. (1975). Organization of Chemical Reactions Into Dividing and Metabolizing Units: The Chemotons. BioSystems, 7, 15-21

Ganti, T. (2003). The Principles of Life. Oxford: Oxford University Press

Glansdorff, P., \& Prigogine, I. (1971). Thermodynamics of Structure, Stability and Fluctuations. London: Wiley 
Hofmeyr, H.-J. S. (2007). The Biochemical Factory that Autonomously Fabricates Itself: A Systems Biological View of the Living Cell. In F. Boogerd, F. J. Bruggerman, J.-H. S. Hofmeyr, \& H. V. Westerhoff (Eds.), Systems Biology: Philosophical Foundations (pp. 217-242). Amsterdam: Elsevier.

Juarrero, A. (2009). Top-down Causation and Autonomy in Complex Systems. In N. Murphy, G. Ellis \& T. O'Connor (Eds.), Downward Causation and the Neurobiology of Free Will (pp. 83-102). Berlin, Heildelberg: Springer-Verlag.

Kant, E. [1781] (1987). Kritik der Urteilkraft (Critique of Judgment. Indianapolis: Hackett Publishing)

Kauffman, S. (2000). Investigations. Oxford: Oxford University Press

Kim, J. (1993). Supervenience and Mind: Selected Philosophical Essays. Cambridge: Cambridge University Press

Kim, J. (1997). Explanation, Prediction and Reduction in Emergentism. Intellectica, 25(2), 45-57

Kim, J. (1998). Mind in a Physical World. Cambridge MA: MIT Press

Kim, J. (2003). Blocking Causal Drainage and Other Maintenance Chores with Mental Causation. Philosophy and Phenomenological Research, 67(1), 151-176

Kim, J. (2006). Emergence: Core Ideas and Issues. Synthese, 151(3), 547-559

Kim, J. (2010). Essays in the Metaphysics of Mind. Oxford: Oxford University Press

Laughlin, R., \& Pines, D. (2000). The Theory of Everything. Proceedings of the National Academy of Science of the United States of America, 97, 28-31

Laughlin, R., Pines, D., Schmalien, J., Stojkovic, B. \& Wolynes, P., (2000). The middle way. Proceedings of the National Academy of Science of the United States of America, 97, 32-37

Lloyd Morgan, C. (1923). Emergent Evolution. London: Williams and Norgate.

Luisi, P.-L. (2006). The Emergence of Life: From Chemical Origins to Synthetic Biology. Cambridge: Cambridge University Press.

Maturana, H., \& Varela, F. (1980). Autopoiesis and Cognition. The Realization of the Living. Dordrecht: Reidel Publishing.

Mayr, E. (2004). What Makes Biology Unique? Considerations on the Autonomy of a Scientific Discipline. Cambridge: Cambridge University Press

McLaughlin, B. P. (1992). The Rise and Fall of British Emergentism. In A. Beckermann, H. Flohr, \& J. Kim (Eds.), Emergence or Reduction? Essays on the Prospects of Nonreductive Physicalism (pp. 49-93). Berlin: Walter de Gruyter.

Mill, J. S. (1843). A System of Logic. London: Parker.

Mossio, M. (2013). Closure. In W. Dubitzky, O. Wolkenhauer, K.-H. Cho, \& H. Yokota (Eds.), Encyclopedia of Systems Biology. New York: Springer.

Mossio, M. \& Moreno, A. (2010). Organizational closure in biological organisms. History and Philosophy of the Life Sciences, 32(2-3), 269-288

Mossio, M., Saborido C. \& Moreno, A. (2009). An Organizational Account of Biological Functions. British Journal for the Philosophy of Science, 60, 813-841

Nicolis, G., \& Prigogine, I. (1977). Self-organization in Nonequilibrium Systems: From Dissipative Structures to Order Through Fluctuations. New York: Wiley \& Sons.

Pattee, H. H. (1972). Laws, Constraints, Symbols and Languages. In C. H. Waddington (Ed.), Towards a Theoretical Biology 4: Essays (pp. 248-258). Edinburgh: Edinburgh University Press.

Pattee, H. H. (1973) (Ed.). Hierarchy Theory. The Challenge of Complex Systems. New York: Georges Braziller.

Piaget, J. (1967). Biologie et Connaissance. Paris: Gallimard.

Rosen, R. (1972). Some Relational Cell Models: The Metabolism-Repair Systems. In R. Rosen (Ed.), Foundations of Mathematical Biology (vol. II, pp. 217-253). New York: Academic Press. 
Rosen, R. (1991). Life Itself: A Comprehensive Inquiry into the Nature, Origin and Fabrication of Life. New York: Columbia University Press.

Ruiz-Mirazo, K. (2001). Physical Conditions for the Appearance of Autonomous Systems with Openended Evolutionary Capacities. PhD Dissertation, University of the Basque Country

Saborido, C., Mossio, M. \& Moreno, A. (2011). Biological Organization and Cross-Generation Functions. The British Journal for the Philosophy of Science, 62, 583-606

Salmon, W. C. (1998). Causality and Explanation. Oxford: Oxford University Press.

Silberstein, M. \& McGeever, J. (1999). The Search for Ontological Emergence. Philosophical Quarterly, 50(195), 182-200

Soto, A. M., Sonnenschein, C. \& Miquel, P. A. (2008). On Physicalism and Downward Causation in Developmental and Cancer Biology. Acta Biotheoretica, 56, 257-274

Sperry, R. W. (1969). A Modified Concept of Consciousness. Psychological Review, 76(6), 532-536

Stephan, A. (1992). Emergence - A Systematic view on its Historical Facets. In A. Beckermann, H. Flohr, \& J. Kim (Eds.), Emergence or Reduction? Essays on the Prospects of Nonreductive Physicalism (pp. 25-48). Berlin: Walter de Gruyter.

Teller (1986). Relational Holism and Quantum Mechanics. The British Journal for the Philosophy of Science, 37, 71-81

Thompson, E. (2007). Mind in life: biology, phenomenology, and the sciences of mind. Cambridge MA: The Belknap Press of Harvard University Press.

Umerez, J. \& Mossio, M. (2013). Constraint. In W. Dubitzky, O. Wolkenhauer, K.-H. Cho, \& H. Yokota (Eds.), Encyclopedia of Systems Biology. New York: Springer.

Van Gulick, R. (2001). Reduction, Emergence and Other Recent Options on the Mind/Body Problem: A Philosophical Overview. Journal of Consciousness Studies, 8(9-10), 1-34

Varela, F. (1979). Principles of Biological Autonomy. New York: North Holland.

Varela, F., Maturana, H., \& Uribe, R. (1974). Autopoiesis: the organization of living systems, its characterization and a model. BioSystems, 5, 187-196

Vicente, A. (2011). Current Physics and 'the Physical'. The British Journal for the Philosophy of Science, 62(2), 393-416

Weber, A., \& Varela, F. (2002). Life after Kant: Natural Purposes and the autopoietic foundations of biological individuality. Phenomenology and the Cognitive Sciences, 1, 97-125 